\title{
Dietary Supplementation with Specific Collagen Peptides Has a Body Mass Index-Dependent Beneficial Effect on Cellulite Morphology
}

\author{
Michael Schunck, Vivian Zague, ${ }^{1}$ Steffen Oesser, and Ehrhardt Proksch ${ }^{3}$ \\ ${ }^{1}$ CRI, Collagen Research Institute, Kiel, Germany. \\ ${ }^{2}$ Department of Cell and Developmental Biology, Institute of Biomedical Sciences, University of São Paulo, São Paulo, Brazil. \\ ${ }^{3}$ Department of Dermatology, Christian-Albrechts-University of Kiel, Kiel, Germany.
}

\begin{abstract}
In this double-blind, placebo-controlled clinical study, we investigated the efficacy of specific bioactive collagen peptides $(\mathrm{BCP})$ on the cellulite treatment of normal and overweight women. In total, 105 women aged 24-50 years with moderate cellulite were randomized to orally receive a daily dosage of $2.5 \mathrm{~g} \mathrm{BCP}$ or a placebo over 6 months. The degree of cellulite was evaluated before starting the treatment and after 3 and 6 months of intake. In addition, skin waviness, dermal density, and the length of subcutaneous borderline were assessed. BCP treatment led to a statistically significant decrease in the degree of cellulite and a reduced skin waviness on thighs $(P<0.05)$ in normal weight women. Moreover, dermal density was significantly improved $(P<0.05)$ compared to placebo. The subcutaneous borderline showed a significant shortening after BCP intake compared to the beginning of the study, indicating cellulite improvement, but the data failed to reach statistical significance compared to placebo. The efficacy of BCP treatment was also confirmed in overweight women, although the impact was less pronounced in comparison with women of normal body weight. The results of the study demonstrated that a regular ingestion of BCP over a period of 6 months led to a clear improvement of the skin appearance in women suffering from moderate cellulite. Based on the current data, it can be concluded that a long-term therapy with orally administered BCP leads to an improvement of cellulite and has a positive impact on skin health.
\end{abstract}

\section{KEY WORDS: $\bullet$ bioactive collagen peptide $\bullet$ body mass index $\bullet$ cellulite $\bullet$ collagen hydrolysate $\bullet$ dermis density $\bullet$ dietary supplement $\bullet$ oral administration $\bullet$ randomized controlled clinical trial}

\section{INTRODUCTION}

C ELLULITE IS A COMPLEX PROBLEM that affects $~ 85 \%$ of women over the age of 20 . It occurs mainly on the thighs, buttocks, and abdomen and is characterized by orange peel or cottage cheese appearance. ${ }^{1}$

Cellulite involves multifactorial etiologies, including genetic predisposition, gender differences, age, ethnicity, diet, sedentary lifestyle, and pregnancy. ${ }^{2,3}$ It is characterized by the presence of excess subcutaneous fat that bulges into the dermis, blood, and lymphatic disturbances and altered dermal extracellular matrix.

Although cellulite is often present in healthy nonobese patients, it is exacerbated by overweight. Clinical grades of cellulite are positively correlated with body mass index (BMI). ${ }^{4}$ Mirrashed et al. ${ }^{4}$ and Hexsel et al. ${ }^{5}$ reported that affected individuals with higher BMIs have a weaker, less

Manuscript received 11 February 2015. Revision accepted 31 August 2015.

Address correspondence to: Ehrhardt Proksch, MD, PhD, Department of Dermatology, Christian-Albrechts-University of Kiel, Schittenhelmstr. 7, Kiel D-24105, Germany, E-mail: eproksch@dermatology.uni-kiel.de dense connective tissue structure, leading to the increased extrusion of adipose tissue lobules into the dermis.

A variety of therapies have been proposed for the cellulite treatment, including weight loss, exercises, massage, and various topical agents, as well as oral supplements and functional foods. ${ }^{2,6,7}$ However, there are few scientifically reported studies examining the extent to which they improve the condition. Successful treatment of cellulite will ultimately depend on an improvement in the understanding of the pathophysiology of cellulite adipose tissue. ${ }^{6}$ It is commonly accepted that dermal and subcutaneous connective tissue, which has been weakened by altered and disordered extracellular matrix, plays a key role in the pathophysiology of cellulite and contributes to the irregular and dimpled appearance of cellulite-affected skin. Consequently, therapies aiming to restore the normal structure of the dermis and subcutaneous tissue can be a meaningful approach to improving the cellulite condition. ${ }^{2,3,6,7}$

Dietary supplementation with collagen peptides has demonstrated a notably stimulatory effect on dermal cellular metabolism, improving the biosynthesis of extracellular matrix proteins and, consequently, restoring dermal structure. 
There is an agreement that biological effects promoted by food-derived collagen peptides are related to the ingestion of collagen in its hydrolyzed form. Collagen peptides are a bioavailable food supplement without any side effects that are derived from native collagen by heat denaturation and further enzymatic hydrolysis. ${ }^{8,9}$ Specific and complex multistep hydrolysis gives rise to biologically active collagen peptides, which have been proven to stimulate dermal metabolism and have positive effects on skin properties. ${ }^{10,11}$

Although it is generally believed that collagen-derived products are hydrolyzed into amino acids in the gastrointestinal tract before being absorbed into the blood circulation system, there is considerable evidence showing that peptides can be absorbed partially intact. ${ }^{9,12}$ Several human and animal bioavailability studies with orally administered collagen peptides have shown that food-derived collagen peptides are absorbed mostly as free amino acids like other protein sources, but also as collagen-derived peptides. ${ }^{9,12-17}$ Therefore, orally ingested collagen peptides are able to cross the intestinal barrier and enter the blood circulation system, from where they are transported to the skin. ${ }^{9,16,17}$ Inside the target connective tissue, collagen peptides affect the metabolic processes of the skin.

Specific bioactive collagen peptides' (BCP) intake stimulated the dermal metabolism of rats by increasing type I and IV collagen expression and reducing MMP-2 activity. ${ }^{10}$ Moreover, Proksch et al. ${ }^{11}$ demonstrated a notable improvement in skin elasticity on healthy women who ingested $2.5 \mathrm{~g} /$ day of specific collagen peptides for 8 weeks. In another clinical trial conducted by Proksch et al. ${ }^{18}$ a significant increase of collagen I and elastin content was observed in the skin of women who consumed the same specific collagen peptides and dosage for 8 weeks.

Based on the previous findings that have demonstrated the efficacy of specific collagen peptides on improving dermal strength and skin elasticity, it can be speculated that the oral supplementation of such peptides may also have a positive impact on cellulite-affected skin.

In this clinical study, for the first time, the influence of specific BCP ingestion on the treatment of cellulite in normal and overweight women was investigated after 6 months of daily intake.

\section{MATERIALS AND METHODS}

\section{$B C P$}

The specific BCP used in this study was composed of collagen peptides derived from a complex multistep procedure of porcine skin type I collagen degradation. The product was provided by GELITA AG, commercially available as VERISOL $^{\circledR}$. The product is clearly defined by a MALDI-MS mass peaks fingerprint with specific collagen peptides of an average molecular weight of $2.0 \mathrm{kDa}$. The product had proven its high safety when administered orally in previous clinical studies. ${ }^{11,12}$

\section{Study design}

The study was carried out as a monocentric, double-blind, randomized, placebo-controlled supplementation study on the effects of specific BCP on the degree of cellulite on thighs and skin surface profile and dermal density after 6 months of daily intake.

The study was approved by the Freiburger EthikKommission International, Freiburg, Germany, adhered to current good clinical practice regulations, and was in accordance with the revised Declaration of Helsinki. All test subjects received detailed information listing of every single parameter relevant to the study. All subjects gave signed informed consent after receipt of the written information and having had a possibility for further questions.

\section{Subjects}

A total of 105 healthy female subjects with moderate cellulite on the thighs (score 2-3 according to a modified score by Nürnberger and Müller ${ }^{19}$ ) were included in the study and randomized for treatment with a daily dose of $2.5 \mathrm{~g}$ of BCP or placebo (maltodextrin). In both treatment groups, the subjects were classified, depending on their BMI, as being of normal weight $(\mathrm{BMI}<25$ ) or being overweight $($ BMI $>25)$.

A daily dosage of $2.5 \mathrm{~g}$ of the product or placebo was taken orally by the participants in accordance with the instructions given by the investigator. The powder was to be dissolved in water or any other liquid.

Before the beginning of oral treatment and data acquisition, there was a preconditioning period of at least 7 days. During this period and throughout the entire length of the study, the subjects had to refrain from using any leave-on products and oily or moisturizing skin cleansing products on the thighs. Moreover, any change of their usual skin cleaning routine on the test sites was prohibited. It was also forbidden to intensively expose the test sites to UV light (sun or solarium). The subjects were not allowed to visit saunas, swimming pools, or do intensive sport on the day before the study visits. Massages or any kind of anticellulite treatment on the test site was prohibited 3 months before study start.

Furthermore, any change in their lifestyle, dietary habits, or sport activities, 2 weeks before study start and the consumption of any additional nutritional supplement or vitamin preparations 3 months before study start were also prohibited. The treatment of the test areas with the following medications was barred before the start and during the study: dermatological therapeutics (6 weeks prior), corticosteroids and antihistamines (4 weeks prior), antiinflammatory drugs and antibiotics (2 weeks prior).

\section{Inclusion criteria}

The inclusion criteria were as follows: healthy females ranging from 25 to 50 years of age (homogeneous distribution between treatment groups), moderate cellulite on the thighs: score 2-3 according to a modified score by Nürnberger and Müller ${ }^{19}$ phototypes I to III (Fitzpatrick scale), in general good health and mental condition, personal informed consents to participate in the study, personal presence on the predefined days at the institute, willing and able to follow the study rules, and a fixed schedule. The criteria 
of inclusion were checked by the investigator before study start. If there had been divergence from these criteria, the subject would not have been included in the data analysis of the study.

\section{Exclusion criteria}

The exclusion criteria were as follows: any deviation from the above-mentioned inclusion criteria, acute skin diseases (e.g., atopic eczema, atopic dermatitis, psoriasis) on the test sites, or other dermatological disorders (scars, sunburn, moles), food allergies against ingredients of the test products, gastrointestinal diseases or indigestion, tattoos on the test sites, topical medication used on the test area within 6 weeks before the start of the study, systemic medication with antiinflammatory agents or antibiotics within 2 weeks before the start, systemic medication with corticoids and/or antihistamines within 4 weeks before the start, medication with other systemic medication within 4 weeks before the start, systemic illness of the subject at the beginning of the study, pregnancy or a period of breast feeding, immunological disorders, severe disorders within 6 months before the start, for example, cancer, acute cardiac and circularity disorders, severe diabetes, alcohol and drug abuse, participation in other studies with cosmetic products on the test areas within 2 weeks before the start or during the study, participation in a study with a pharmaceutical preparation within 4 weeks before the start, intake of nutritional supplements within 4 weeks before the start and, except for the test products administered during the study, change in lifestyle or eating habits during the study, treatment with leave-on products and oily or moisturizing skin cleansing products on the thighs, or a change in the usual skin care routine, massage or any kind of anticellulite treatment on the test site 3 months before study start and during the study exposure to intensive sunlight or artificial UV (solarium) on the test sites within 1 week before the start, swimming, sauna or intensive sport within 1 day before measurements, lack of compliance, intellectual or mental inability to follow study instructions.

\section{Assessments}

Test sites. All tests were performed in a defined area on the thighs. For the cellulite degree assessment (pinch test), the left thigh and buttock were used. Skin waviness and dermal density were performed on the left thigh.

On every measurement day, the subjects had to expose their uncovered test sites to the indoor climatic conditions $\left(21.5^{\circ} \mathrm{C} ; 50 \%\right.$ relative humidity) for at least 30 minutes.

Measurement times. There were three measurement times for all test parameters. Data were collected at the beginning of the study before the product treatment (t0), after 3 months (t1), and after 6 months (t2) of a daily product intake.

Compliance. The subjects were instructed to enter each product intake into the diary. Compliance and tolerance were checked after 1 and 4 weeks and 3 and 5 months of intake and at each measurement time at the institute $(\mathrm{t} 1=3$ months; $\mathrm{t} 2=6$ months) by checking the study diary, dosage, dietary habits, and by questioning the subjects for potential intake gaps and misses.

If there had been a deviation in weight from the baseline measurement of $\pm 5 \%$, the subject would have been excluded from the study. On each compliance check and measurement day, the subjects were asked again if they had adhered to all the restrictions and instructions. Had there been any deviation from the restrictions, this subject would have been excluded from the study.

Measurement of cellulite degree (pinch test). The grade of cellulite was visually/tactilely assessed on the test site by the so-called pinch test. In brief, the assessor pinched the skin on the outer thigh between her index finger and her thumb, evaluated the visible dimpling, and graded cellulite appearance. The grade of cellulite was defined according to a modified score by Nürnberger and Müller ${ }^{19}$ (Table 1).

Measurement of the skin surface profile (waviness, Wt) with PRIMOS ${ }^{\circledR}$ Pico. The skin surface profile (parameter $\mathrm{Wt}$, waviness) was measured in vivo on the thighs using the optical measuring instrument PRIMOS Pico (GFM). The device allows detailed 3D skin surface scanning and provides high-resolution assessment of skin surfaces by using phase-shifted light stripes projected by micromirrors to generate a 3D profile of the measured skin surface (area $18 \times 13 \mathrm{~mm}^{2}$ ). The reflected light is captured by a highresolution camera, and a software package converts the image into a color-coded picture of the skin surface, with different colors for different heights.

The measurements were performed on the left thigh on a site prior marked with a permanent marker, while the subjects were standing. Three pictures were taken per test site. Height images were computed according to the standard procedure using mathematical filters. These height images were used to calculate star roughness. For the calculation of star roughness, intersections are arranged in a star shape by the program. Three stars with different locations of their

Table 1. Grade of Cellulite, According TO A Modified Score by Nürnberger AND MülleR ${ }^{19}$

\begin{tabular}{ll}
\hline Score & \multicolumn{1}{c}{ Description } \\
\hline $0-<0.5$ & $\begin{array}{l}\text { No cellulite } \\
0.5-1.5\end{array}$ \\
& $\begin{array}{l}\text { Smooth surface of skin while lying down and standing } \\
\text { Mattress phenomenon upon pinch test }\end{array}$ \\
$>1.5-2.5$ & $\begin{array}{l}\text { Smooth surface of skin while lying down } \\
\text { Dimpling at the inner and outer sides of the thigh and at } \\
\text { the back of the thigh while standing } \\
\text { Mattress phenomenon clearly visible upon pinch test }\end{array}$ \\
$>2.5-3.5$ & $\begin{array}{l}\text { Slightly dimpled surface of skin while lying down } \\
\text { Dimpling at the entire thigh and buttock while standing } \\
\text { Mattress phenomenon very clearly visible upon pinch test }\end{array}$ \\
$>3.5-4$ & $\begin{array}{l}\text { Severely dimpled surface of skin at thigh and buttock } \\
\text { while lying down and standing. Belly and upper arms } \\
\text { may be affected too. }\end{array}$ \\
\hline
\end{tabular}


central point were defined. The calculation of the parameter occurs according to the determination of the line roughness (separate for every star shape-arranged intersection).

The parameter waviness $(\mathrm{Wt})$ was analyzed. It represents the sum of the height of the largest profile peak and the largest profile valley of the waviness profile. It is presented in millimeter. The mean of the three single measurements was calculated for each test site and point in time.

Dermis density and length of borderline between dermis and subcutis by ultrasound (DermaScan ${ }^{\circledR} C$ ). At each measurement point in time, dermis density and the length of the borderline between dermis and subcutis were measured using the DermaScan C $20 \mathrm{MHz}$ ultrasound device and the DSCANV3 application software (Cortex Technology). The device was operated according to the manufacturer's instructions.

The probe was configured with a $20 \mathrm{MHz}$ transducer, which offers a $60 \times 130 \mu \mathrm{m}$ resolution and a $10 \mathrm{~mm}$ penetration depth. Before use, the ultrasound probe was prepared for scanning by mounting a plastic membrane and injecting distilled water into the probe. Finally, a small amount of gel was applied into the open slit of the gel spacer.

Three single B-mode scans on each test site were conducted, using a defined gain profile that was adjusted to the depth and density distribution in the tissue being scanned (thigh). This gain profile was the same for all subjects. The gain level was adjusted for each subject, but was the same for all measurements performed on this subject for all points in time. Adjusting the gain level makes it possible to compensate for the attenuation of the ultrasound signal through the tissue. Signals are amplified by the gain as a function of depth.

Echo-free structures such as fat and lymphatic fluid are displayed as black regions in the ultrasound images, while connective tissue structures appear in green, red, or yellow. Because the ultrasound reflection intensity is related to the relative density of the targeted tissue, it also provides information on the arrangement of the collagen and elastic fibers. Echogenicity reflects dermal extracellular matrix organization; that is, the more organized and abundant the collagen tissue is, the higher the echogenicity.

The borderline between dermis and subcutis (line 2) was defined using the freehand function. The length of the defined line was measured automatically by the software. The measurement values were presented in millimeter. The length is a measure for the degree of cellulite. The wavier the line due to protrusion of adipose tissue into the dermis (papillae adipose), the longer the line is and the more pronounced the cellulite is.

\section{Statistical analysis}

Statistically significant differences in age, body weight, BMI, and grade of cellulite at baseline between both treatment groups were analyzed by the Mann-Whitney $U$-Test for all participants and considering normal and overweight subdivision. For a $P$-value $<0.05$, the difference was accepted as statistically significant.
Statistically significant changes in the grade of cellulite within the treatment groups were calculated with the Wilcoxon signed-rank test in comparison to the baseline situation. For a $P$-value $<0.05$, the difference was accepted as statistically significant.

Changes within the treatment groups for the other measurement parameters, such as skin waviness, dermal density, and length of borderline, were tested with the Wilcoxon signed-rank test in comparison to the baseline situation. Differences between both treatment groups were analyzed with the Student's $t$-test for two independent samples. For a $P$-value $<0.05$, the difference was accepted as statistically significant.

\section{RESULTS}

\section{Subjects and dropouts}

A total of 105 women between 24.8 and 53.5 years of age $(40.6 \pm 7.4)$ were initially included in the study. Ninetyseven of the 105 subjects finished the study correctly, representing the evaluate per-protocol population. At baseline, no statically significant differences between the treatment group and the placebo group (Table 2, $P=0.529$ ) could be observed.

During the study period, five subjects dropped out (one placebo, four BCP) due to private reasons and three subjects (two placebo, one BCP) dropped out because of lacking compliance or illness.

None of the dropouts was product related, and no side effects or discomfort were observed during the study. No adverse reactions were recorded in any of the study participants.

\section{Study groups}

The results of 105 subjects (53 in the BCP group, 52 in the placebo group) were included in the data analysis

Table 2. Demographic Data of Study Population Per Treatment Group

\begin{tabular}{lcc}
\hline & $B C P$ & Placebo \\
\hline Total $(n)$ & 53 & 52 \\
Cellulite score & $2.4 \pm 0.4$ & $2.4 \pm 0.4$ \\
Normal BMI $\leq 25(n)$ & 24 & 26 \\
Age (years) & $39.9 \pm 7.2$ & $39.0 \pm 6.5$ \\
Weight $(\mathrm{kg})$ & $61.8 \pm 5.1$ & $62.0 \pm 4.7$ \\
BMI & $22.2 \pm 1.2$ & $22.4 \pm 1.1$ \\
Cellulite score & $2.2 \pm 0.3$ & $2.3 \pm 0.4$ \\
Overweight BMI $>25(n)$ & 25 & 22 \\
Age (years)* & $38.4 \pm 6.5$ & $42.6 \pm 6.6$ \\
Weight $(\mathrm{kg})$ & $74.8 \pm 6.7$ & $76.3 \pm 6.1$ \\
BMI & $26.5 \pm 1.6$ & $26.7 \pm 1.5$ \\
Cellulite score & $2.5 \pm 0.1$ & $2.6 \pm 0.1$ \\
\hline
\end{tabular}

No statistically significant differences in age, body weight, and BMI between both treatment groups were determined at study baseline. Only in the overweight subgroup a difference in age was observed $(* P<0.05$, mean \pm SD, $n=$ number of subjects).

$\mathrm{BCP}$, bioactive collagen peptides; BMI, body mass index. 
Table 3. The Cellulite Score in the Overall Study Population and Normal and Overweight Women, Before and After Oral Supplementation with Specific Bioactive Collagen Peptide in Comparison with Placebo Group

\begin{tabular}{|c|c|c|c|c|c|}
\hline$B M I$ & Treatment & $\mathrm{n}$ & Baseline & 3 Months & 6 Months \\
\hline \multirow[t]{2}{*}{ Total study population } & $\mathrm{BCP}$ & 49 & $2.37 \pm 0.4$ & $2.17 \pm 0.5^{*, \#}$ & $2.08 \pm 0.4^{*, \#}$ \\
\hline & Placebo & 48 & $2.44 \pm 0.4$ & $2.23 \pm 0.5^{*}$ & $2.19 \pm 0.5^{*}$ \\
\hline \multirow[t]{2}{*}{ Normal (BMI <25) } & $\mathrm{BCP}$ & 24 & $2.19 \pm 0.3$ & $1.96 \pm 0.4$ & $1.86 \pm 0.4^{*, \#}$ \\
\hline & Placebo & 26 & $2.31 \pm 0.4$ & $2.07 \pm 0.5^{*}$ & $2.04 \pm 0.5^{*}$ \\
\hline \multirow[t]{2}{*}{ Overweight $(\mathrm{BMI}>25)$} & $\mathrm{BCP}$ & 25 & $2.54 \pm 0.5$ & $2.37 \pm 0.4^{*}$ & $2.30 \pm 0.4^{*, \#}$ \\
\hline & Placebo & 22 & $2.59 \pm 0.4$ & $2.40 \pm 0.6^{*}$ & $2.40 \pm 0.5^{*}$ \\
\hline
\end{tabular}

*Intragroup comparison, $P<0.05$ versus baseline.

\#Intergroup comparison, $P<0.05$ versus placebo after 3 and 6 months treatment, mean $\pm \mathrm{SD}, n=$ number of subjects).

(ITT population). At baseline, study groups were balanced regarding the age body weight, BMI, and grade of cellulite showing no statistically significantly differences between both treatment groups (Table 2). Only in the study group representing participants with a BMI $>25$ was an inhomogeneous distribution of the age observed $(P<0.05)$.

\section{Cellulite degree assessment (pinch test)}

After 3 months of BCP treatment, a decrease in the cellulite score could be observed $(2.17 \pm 0.5)$ compared to the baseline situation $(2.37 \pm 0.4)$. This statistically significant decrease $(P<0.05)$ continued for the next 3 months, reaching a maximal score decrease of 0.3 score points at the end of the study $(P<0.05)$ (Table 3$)$. A clear improvement compared to the placebo group was detected. After 3 and 6 months of BCP treatment, a statistical and significant reduction of the cellulite score was determined $(P<0.05)$.

In the normal BMI $(\leq 25)$ study group, the data revealed a decreased cellulite score of $5.3 \%$ compared to placebo after 3 months of treatment. At the end of the study at 6 months, the observed effect was even more pronounced reaching a mean reduction in the score of about $9 \%$ compared to placebo (Table 3). These findings reflected a clear attenuation of mattress phenomenon upon the pinch test, a smoother surface of the thighs while lying down, and a decrease in the dimpling of the thigh while standing. This improvement could also be confirmed in the group of women with a BMI $>25$, although the beneficial effect after 6 months of treatment was less pronounced. In this study, a score reduction of $4 \%$ could be observed in comparison to the untreated women (Table 3 ).

\section{Skin surface profile measurement (waviness)}

In examinations of the skin surface profile, it could be demonstrated that the relative waviness of the skin in the women treated with $\mathrm{BCP}$ was reduced by $2 \%$ compared to the placebo group after 3 months. This trend was even more pronounced after 6 months of BCP intake. Compared to the placebo group, a statistically significant reduction $(P<0.05)$ of $8 \%$ in skin waviness could be observed after 6 months of treatment (Fig. 1). There was a noticeable improvement in skin waviness promoted by $\mathrm{BCP}$, which can be seen in the representative 3D colored images of Figure 2, in which each color is related to a height. The cellulite-affected area in both placebo (Fig. 2A) and BCP (Fig. 2B) groups, at baseline, showed a typical skin profile with marked red and yellow areas, indicating the skin depression and relief as for skin surface, respectively. In contrast, a remarkable improvement of skin depression with attenuated red areas and

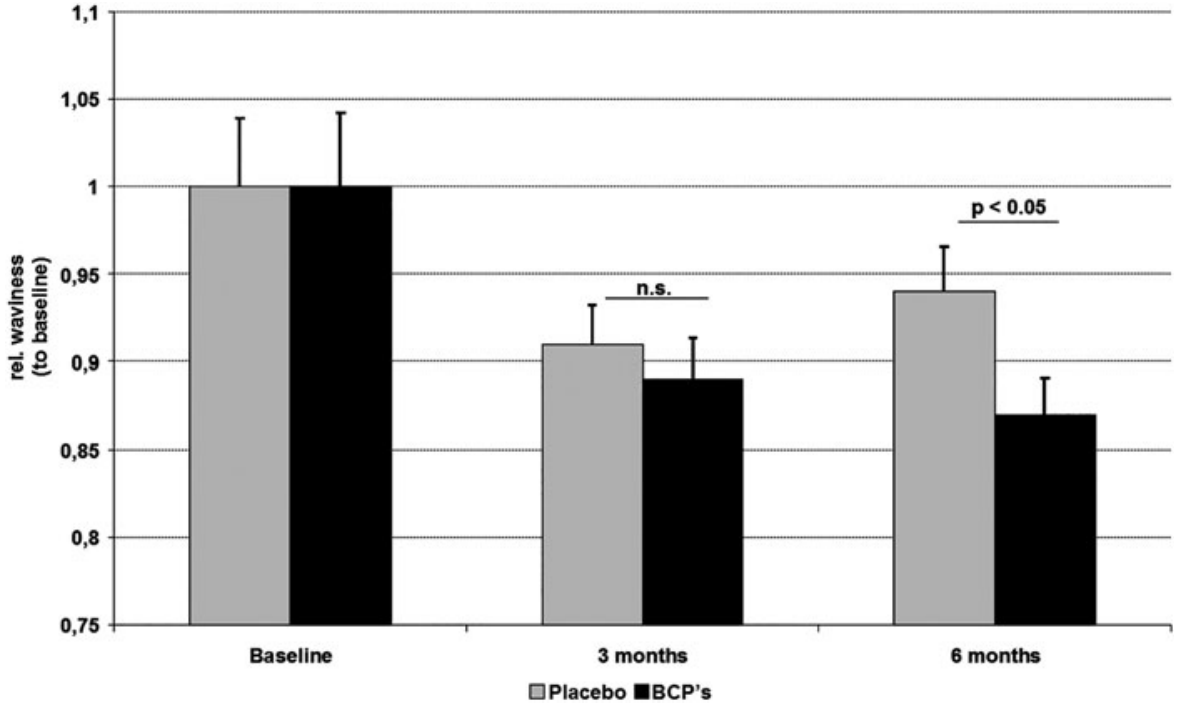

FIG. 1. Skin surface profile of tight skin before and after 3 and 6 months of oral supplementation with bioactive collagen peptide (BCP) or placebo, measured by PRIMOS ${ }^{\circledR}$ Pico. Skin waviness of thigh was statistically significantly decreased $(P<0.05)$ after 6 months of BCP daily intake in the overall study subjects in comparison with placebo treatment (mean \pm standard error of mean, n.s. not statistically significant). 

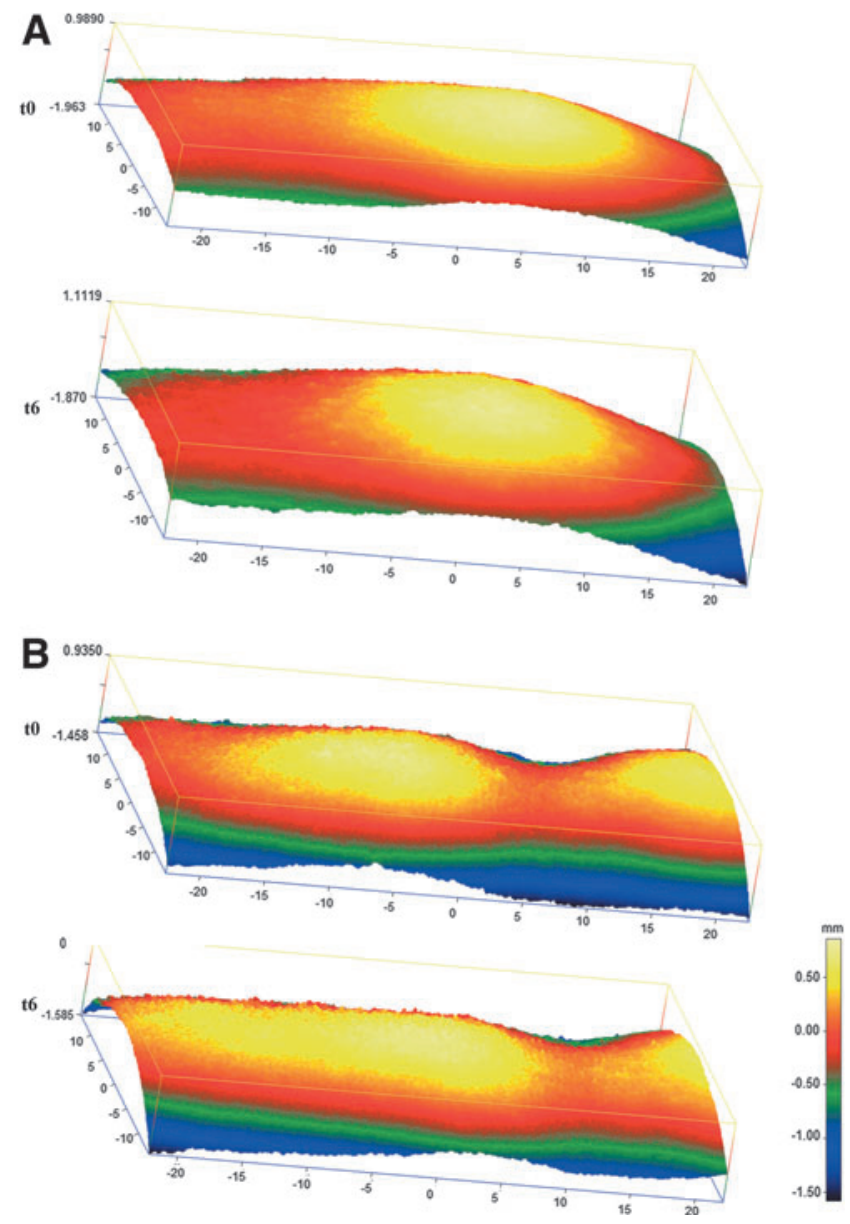

FIG. 2. Representative 3D color-coded height images of tight skin surface, before (t0) and after 6 months (t6) of oral supplementation with BCP or placebo; measured by PRIMOS Pico. Each color is related to a height with red and yellow areas, indicating the skin depression and relief as for skin surface, respectively. At the external margins of the measuring fields, the resolution is limited seen by lower color intensity in green and blue. (A) Placebo group. (B) BCP group. A notable improvement in skin waviness can be seen in the BCP group, showing more homogeneous skin surfaces.

a more homogeneous skin surface was observed in the BCP group after 6 months (Fig. 2B), while the placebo group did not change visibly (Fig. 2A).

In women with a maximum BMI of 25 , a statistically significant improvement could already be observed after 3 months of BCP intake compared to the beginning of the study $(P<0.01)$. Compared to the placebo group, waviness was significantly reduced $(P<0.05)$ after BCP treatment. At the end of the study (6 months), a pronounced decrease in waviness of $11.1 \%$ could be detected. This improvement in the skin appearance was statistically significant compared to placebo $(P<0.05)$.

In overweight participants $(\mathrm{BMI}>25), \mathrm{BCP}$ treatment also led to a skin waviness reduction of $3.6 \%$ after 6 months in comparison to placebo, but the data failed to reach the level of statistical significance.

\section{Dermis density measurement}

Evident improvement in dermal density owing to 6 months of BCP intake can be visualized by the ultrasound representative images in Figure 3A. At baseline, for both the placebo and $\mathrm{BCP}$ groups, the interface between the dermis and subcutis appeared as a broken irregular line, a high amount of black areas (fat cells and lymphatic fluid), and a low degree of dermal density (green areas). After 6 months of BCP treatment, the skin tissue becomes measurably more compact, indicating a strengthening of connective tissue, with echo-free interspaces (black areas) being reduced and clearly high dermal density (red and yellow areas).

Measurement of the dermis density demonstrated a progressive loss in density over 6 months in all women assigned to the placebo group (6.85-6.45). This statistically significant $(P<0.01)$ decrease of $3.1 \%$ could not be observed in

A
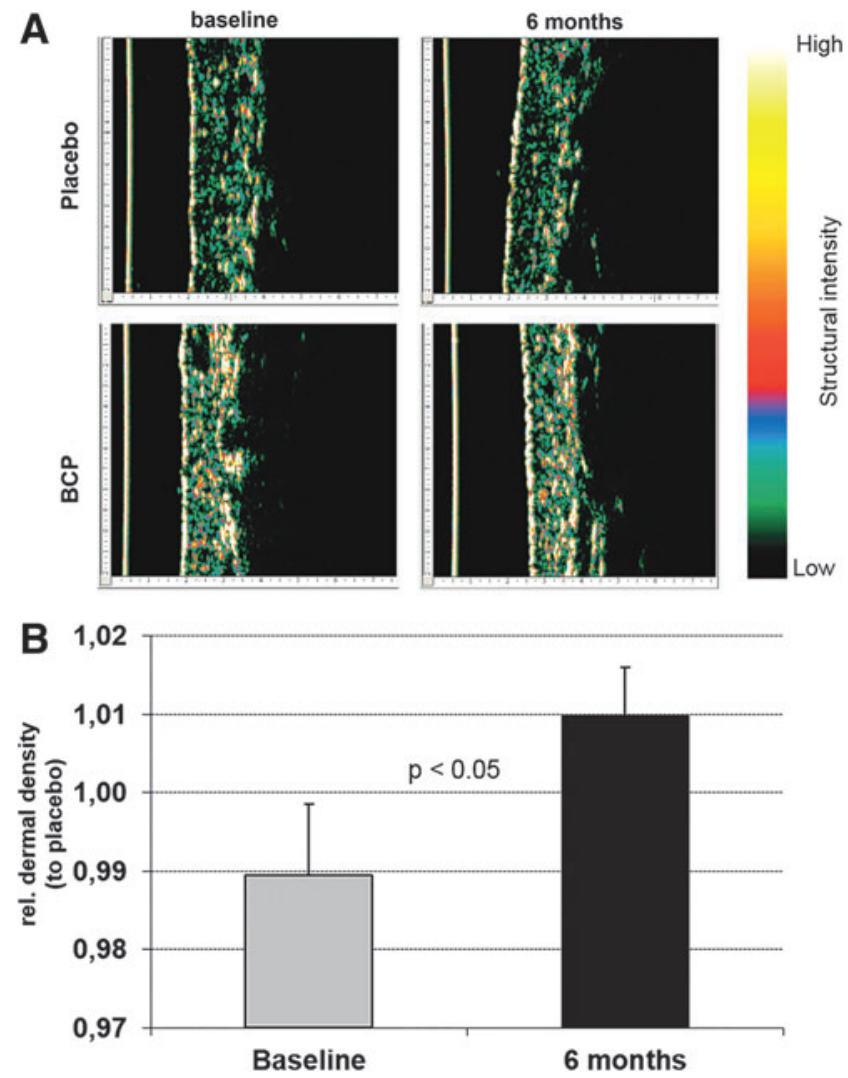

FIG. 3. Dermis density of cellulite-affected area in tights after 6 months of BCP or placebo intake, measured by DermaScan. (A) Representative ultrasound cross-sectional images (single B scan) of $\mathrm{BCP}$ and placebo groups. The epidermis of the skin pictures is left located (yellow line). The dermis connective tissues are showing several degrees of dermal density (green low and red to yellow high density). Black structures are fat and lymphatic fluid. Note that before therapy (baseline), the interface between the dermis and the subcutis appears as a broken and irregular line and many black areas appear. After 6 months of BCP daily supplementation, dermis tissue has become measurably more compact, indicating a strengthening of the connective tissue. Black interspaces have been reduced. (B) Relative dermis density of BCP group to placebo, showing an increased dermal density after BCP treatment (7.29-7.66). 
the $\mathrm{BCP}$ group. Overall, data revealed that a $\mathrm{BCP}$ treatment over 6 months led to a statistically significant $(P<0.05)$ increased dermal density compared to placebo (Fig. 3B).

\section{Measurement of the borderline length between dermis and subcutis}

Examinations of the borderline length between dermis and subcutis showed a clear statistically significant $(P<0.05)$ shortening of the borderline after a BCP intake over 6 months compared to the beginning of the study (data not shown). As the length of the borderline correlates with the degree of cellulite, the data demonstrated a statistically significant improvement of cellulite after BCP treatment. Further evaluation of the data, including the results representing normal and overweight women, failed to reach level of statistical significance in comparison with the placebo.

\section{DISCUSSION}

The term cellulite is often used to describe a complex architectural disorder of the skin with multifactorial etiologies. ${ }^{3}$ In general, the formation of cellulite is a natural process and not a disease, reflected by an incidence of about $85 \%$ in adult females. However, the appearance of cellulite can negatively affect the quality of life in women and some seriously suffer from their dimpling orange peel skin.

The pathophysiology of cellulite is not yet fully understood, but it is generally accepted that, beside an altered connective tissue structure and a disturbed dermal extracellular matrix, overweight also has a negative effect on cellulite-prone skin areas such as thighs and buttocks. Mirrashed et al. ${ }^{4}$ demonstrated a correlation between overweight and cellulite pathophysiology in a pilot study with magnetic resonance imaging technique. Even though cellulite formation is exacerbated in combination with being overweight, it is predominantly dependent on the perpendicular orientation and status of dermal septae with regard to the skin surface. These dermal septae are much thinner and more radially oriented in cellulite-affected females compared to unaffected men and women. ${ }^{20}$

Today, a wide variety of cellulite treatment options exist, ${ }^{21}$ ranging from topically applied products and massages to laser and intense pulsed light treatment among others. ${ }^{1}$ Recently, an oral supplementation with polyphenol-rich chokeberry juice taken over a period of 90 days improved cellulite with a marked reduction in the subcutaneous tissue thickness and edema. ${ }^{22}$ Most therapeutic approaches focus on the induction of lipolysis. However, the efficacy of such cellulite treatments seems to be less than completely satisfying, as reported by Wanner and Avram. ${ }^{23}$

In contrast to these various forms of treatment, an oral therapy with $\mathrm{BCP}$ is based on the premise that specific collagen peptides can improve and increase dermal firmness and skin elasticity from the inside.

It is known that the improvements of dermal strength and skin elasticity are two important therapeutic aims in cellulite treatment. ${ }^{21}$ Incidentally, Ortonne et al..$^{24}$ observed that, regardless of age, the presence of cellulite corresponds to a thinning of the dermal layer, a greater length of the dermis-hypodermis interface, and a decrease of dermal density and biomechanical parameters of skin elasticity and extensibility that are negatively affected by the degree of involvement of cellulite. The authors argue that women with cellulite exhibit skin characteristics typical of older ages.

In addition, Dobke et al. ${ }^{25}$ observed that women without cellulite had a better skin quality (firmer, with less compliance, laxity, and capacity of deformation) in the upper posterior part of the thigh. In contrast, women with cellulite showed greater laxity and weakness of the dermis and connective tissue, which extended into the superficial fascia.

The efficacy of the BCP used in this study (VERISOL) on skin health and dermal connective tissue metabolism was recently demonstrated in two monocentric, double-blinded, randomized, placebo-control clinical trials on healthy women. Proksch et al. demonstrated that skin elasticity increased statistically significantly by up to $30 \%$ (average $7 \%$ ) after 8 weeks of oral BCP ingestion in comparison to placebo treatment. ${ }^{11}$ In addition, in a follow-up study, an elastic improvement of the skin resulting from an increased biosynthesis of collagen and elastin in the dermal connective tissue was shown. ${ }^{18}$ Hundred and fourteen women aged 45-65 years received $2.5 \mathrm{~g} \mathrm{BCP}$ or placebo once daily. After 8 weeks of intake, a statistically significant higher content of new generated procollagen type I $(65 \%)$ and tropoelastin $(18 \%)$ in interstitial skin fluid compared to the placebo treatment was observed.

In the present study, it could be demonstrated that a daily dosage of $2.5 \mathrm{~g} \mathrm{BCP}$ has a positive impact on women affected by moderate cellulite. Beside a significant improvement in the cellulite score, results also revealed a significant decrease in the waviness of the thigh skin as well as dermis density strengthening after BCP treatment.

With regard to the mode of action, it could be speculated that the efficacy of BCP on cellulite treatment is based on its positive impact on dermal connective tissue synthesis. This assumption was confirmed by the results of the dermal density measurements. After 6 months of oral BCP intake, a statistically significant higher dermal density $(P<0.01)$ could be detected compared to placebo.

One of the reasons for the dimpling effect in skin seems to be an abnormal pattern of distribution of elastic and collagen fibers in dermal septae. ${ }^{26}$

In cellulite-prone skin, both fiber types are unevenly located and partly clumped and, contrarily, in other parts of the dermal septae, rare and thinly distributed. The irregular strength of the connective tissue septae is considered as the most important distinguishing characteristic between cellulite-prone skin and unaffected skin. ${ }^{3}$ It is known that fibrillin, as the main component of elastic fibers, is of particular importance for the integrity of these fiber bundles. ${ }^{27}$ In a previous study, it could be demonstrated that, beside type I collagen and elastin, the fibrillin syntheses were also affected by the oral administration of BCP (VERISOL). ${ }^{18}$ This increased synthesis of the fiber matrix molecules became clinically apparent in a statistically significant reduction of eye wrinkle deepness of $20 \%$ compared to the placebo group. 
Based on these findings, it could be speculated that the improvement in skin waviness in cellulite-prone skin observed here might be caused by the same effect. In comparison to the control group, a statistically significantly reduced skin waviness $(-11 \%)$ was detected on thighs in normal weight volunteers after BCP administration.

The efficacy of a BCP treatment could also be confirmed in overweight women $(\mathrm{BMI}>25)$, although the impact in this group was less pronounced in comparison to the results in women of normal weight. This observation was reflected by almost all the results obtained from the different investigations.

It could be speculated that, especially in the group of overweight women, the duration of the treatment might be too short to see the maximum effect, as the data revealed an improvement trend but failed to reach statistical significance. It has to be stated that, at baseline, the degree of cellulite in the overweight women was statistically significantly $(P<0.01)$ more pronounced than with women of normal weight, so that an extended therapy might be useful.

Moreover, as the therapeutic effect of a BCP treatment seems to predominantly influence the dermal matrix synthesis and, thus, strengthen dermal connective tissue, the observed effect might be more distinct when the cellulite is specifically caused by connective tissue alterations. This may support the hypothesis that BCP treatment particularly influences the positive impact on fiber septae regeneration. The evaluation of the ultrasound images documents a visually detectable change in tissue structure. It was observed that the network of collagen/elastic fibers in the dermis and subcutis becomes denser.

It has been shown that noninvasive measures such as skin profile and those obtained from ultrasound images exhibit a positive correlation among them and the subjective perception of cellulite. ${ }^{28}$ Dermal thickness may be reduced when it is more compact, and the dermal/hypodermal interface may be shortened when the invagination of adipose tissue toward the deep dermis is reduced as in the slight degree of cellulite. ${ }^{29,30}$ Ultrasound images captured before treatment clearly represented the irregular broken line between dermis and hypodermis, and hypoechoic structures can be interpreted as fat bulging from subcutaneous tissue into the upper-layer dermal tissue. After treatment with BCP, a significant reduction in dermal thickness, elevated echogenicity indicating increased and organized collagen tissue, and reduction of hypoechoic areas were demonstrated.

The results obtained in this study demonstrated that an oral supplementation with specific BCP over a period of 6 months led to a clear improvement of the skin appearance in women suffering from moderate cellulite. In addition, the data showed the marked potential of BCP to improve the skin morphology of cellulite-affected areas, providing new evidence of BCP's beneficial effects and postulating a new therapy strategy for cellulite treatment.

\section{AUTHOR DISCLOSURE STATEMENT}

V.Z. is an employee of GELITA Brazil Ltd. No competing financial interests exist for S.O., M.S., and E.P.

\section{REFERENCES}

1. Rawlings AV: Cellulite and its treatment. Int J Cosmet Sci 2006; 28:175-190.

2. Khan MH, Victor F, Rao B, Sadick NS: Treatment of cellulite: Part II. Advances and controversies. J Am Acad Dermatol 2010;62:373-384.

3. Khan MH, Victor F, Rao B, Sadick NS: Treatment of cellulite: Part I. Pathophysiology. J Am Acad Dermatol 2010;62:361-370.

4. Mirrashed F, Sharp JC, Krause V, Morgan J, Tomanek B: Pilot study of dermal and subcutaneous fat structures by MRI in individuals who differ in gender, BMI, and cellulite grading. Skin Res Technol 2004;10:161-168.

5. Hexsel DM, Abreu M, Rodrigues TC, Soirefmann M, do Prado DZ, Gamboa MM: Side-by-side comparison of areas with and without cellulite depressions using magnetic resonance imaging. Dermatol Surg 2009;35:1471-1477.

6. Hexsel D, Soirefmann M: Cosmeceuticals for cellulite. Semin Cutan Med Surg 2011;30:167-170.

7. Hexsel D, Orlandi C, Zechmeister do PD: Botanical extracts used in the treatment of cellulite. Dermatol Surg 2005;31:866-872.

8. Nemati M, Oveisi MR, Abdollahi H, Sabzevari O: Differentiation of bovine and porcine gelatins using principal component analysis. J Pharm Biomed Anal 2004;34:485-492.

9. Oesser S, Adam M, Babel W, Seifert J: Oral administration of (14)C labeled gelatin hydrolysate leads to an accumulation of radioactivity in cartilage of mice (C57/BL). J Nutr 1999;129:1891-1895.

10. Zague V, de Freitas V, da Costa RM, de Castro GA, Jaeger RG, Machado-Santelli GM: Collagen hydrolysate intake increases skin collagen expression and suppresses matrix metalloproteinase 2 activity. J Med Food 2011;14:618-624.

11. Proksch E, Segger D, Degwert J, Schunck M, Zague V, Oesser S: Oral supplementation of specific collagen peptides has beneficial effects on human skin physiology: A double-blind, placebocontrolled study. Skin Pharmacol Physiol 2014;27:47-55.

12. Liu D, Nikoo M, Boran G, Zhou P, Regenstein JM: Collagen and gelatin. Annu Rev Food Sci Technol 2015;6:527-557.

13. Iwai K, Hasegawa T, Taguchi Y, Morimatsu F, Sato K, Nakamura $\mathrm{Y}$, et al:: Identification of food-derived collagen peptides in human blood after oral ingestion of gelatin hydrolysates. J Agric Food Chem 2005;53:6531-6536.

14. Ohara H, Matsumoto H, Ito K, Iwai K, Sato K: Comparison of quantity and structures of hydroxyproline-containing peptides in human blood after oral ingestion of gelatin hydrolysates from different sources. J Agric Food Chem 2007;55:1532-1535.

15. Sugihara F, Inoue N, Kuwamori M, Taniguchi M: Quantification of hydroxyprolyl-glycine (Hyp-Gly) in human blood after ingestion of collagen hydrolysate. J Biosci Bioeng 2012;113:202-203.

16. Watanabe-Kamiyama M1, Shimizu M, Kamiyama S, Taguchi Y, Sone H, Morimatsu F, Shirakawa H, Furukawa Y, Komai M: Absorption and effectiveness of orally administered low molecular weight collagen hydrolysate in rats. J Agric Food Chem 2010;58:835-841.

17. Kawaguchi T, Nanbu PN, Kurokawa M: Distribution of prolylhydroxyproline and its metabolites after oral administration in rats. Biol Pharm Bull 2012;35:422-427.

18. Proksch E, Schunck M, Zague V, Segger D, Degwert J, Oesser S: Oral intake of specific bioactive collagen peptides reduces skin wrinkles and increases dermal matrix synthesis. Skin Pharmacol Physiol 2014;27:113-119.

19. Nurnberger F, Muller G: So-called cellulite: An invented disease. J Dermatol Surg Oncol 1978;4:221-229. 
20. Querleux B, Cornillon C, Jolivet O, Bittoun J: Anatomy and physiology of subcutaneous adipose tissue by in vivo magnetic resonance imaging and spectroscopy: Relationships with sex and presence of cellulite. Skin Res Technol 2002;8:118-124.

21. Proebstle TM: [Cellulite]. Hautarzt 2010;61:864-872.

22. Ŝavikin K, Menković N, Zdunić G, et al.: Dietary supplementation with polyphenol-rich chokeberry juice improves skin morphology in cellulite. J Med Food 2014;17:582-715.

23. Wanner M, Avram M: An evidence-based assessment of treatments for cellulite. J Drugs Dermatol 2008;7:341-345.

24. Ortonne JP, Zartarian M, Verschoore M, et al.: Queille-Roussel C, Duteil L. Cellulite and skin ageing: Is there any interaction? $J$ Eur Acad Dermatol Venereol 2008;22:827-834.

25. Dobke MK, Dibernardo B, Thompson RC, et al.: Assessment of biomechanical skin properties: Is cellulitic skin different? Aesthet Surg J 2002;22:260-266.
26. Pierard GE, Nizet JL, Pierard-Franchimont C: Cellulite: From standing fat herniation to hypodermal stretch marks. Am J Dermatopathol 2000;22:34-37.

27. Lee J-H, Seo J-H, Park Y-H, Lim K-M, Lee S-J: The effect of hydroxyproline and pro-hyp dipeptide on UV-damaged skin of hairless mice. Korean J Food Sci Technol 2008;40:436-442.

28. Soares JL, Miot HA, Sanudo A, Bagatin E: Cellulite: Poor correlation between instrumental methods and photograph evaluation for severity classification. Int J Cosmet Sci. 2015;37:134-140.

29. Mlosek RK, Debowska RM, Lewandowski M, et al.: Imaging of the skin and subcutaneous tissue using classical and highfrequency ultrasonographies in anti-cellulite therapy. Skin Res Technol 2011;17:461-468.

30. Jasaitiene D, Valiukeviciene S, Linkeviciune G, et al.: Principles of high-frequency ultrasonography for investigation of skin pathology. J Eur Acad Dermatol Venereol 2011;25:375-382. 